\title{
Candidate powdery mildew resistance gene in wheat landrace cultivar Hongyoumai discovered using SLAF and BSR-seq
}

\author{
Junmei Wang ${ }^{1}$, Yahong Li ${ }^{1}$, Fei Xu' ${ }^{1}$, Hongxing $X^{2}{ }^{2}$, Zihang Han ${ }^{1}$, Lulu Liu and Yuli Song ${ }^{1 *}$
}

\begin{abstract}
Background: Wheat powdery mildew, caused by Blumeria graminis f. sp. tritici (Bgt), is an important disease affecting wheat production. Planting resistant cultivars is an effective, safe, and economical method to control the disease. Map construction using next-generation sequencing facilitates gene cloning based on genetic maps and high-throughput gene expression studies. In this study, specific-locus amplified fragment sequencing (SLAF) was used to analyze Huixianhong (female parent), Hongyoumai (male parent) and two bulks (50 homozygous resistant and 50 susceptible $\mathrm{F}_{2: 3}$ segregating population derived from Huixianhong $\times$ Hongyoumai to determine a candidate gene region for resistance to powdery mildew on the long arm of chromosome 7B in wheat landrace Hongyoumai. Gene expressions of candidate regions were obtained using bulked segregant RNA-seq in 10 homozygous resistant and 10 susceptible progeny inoculated by Bgt.. Candidate genes were obtained using homology-based cloning in two parents.

Results: A $12.95 \mathrm{Mb}$ long candidate region in chromosome 7BL was identified, and five blocks in SLAF matched the scaffold of the existing co-segregation marker Xmp 1207. In the candidate region, 39 differentially expressed genes were identified using RNA-seq, including RGA4 (Wheat_Chr_Trans_newGene_16173)—a disease resistance protein whose expression was upregulated in the resistant pool at $16 \mathrm{~h}$ post inoculation with Bgt. Quantitative reverse transcription (qRT)-PCR was used to further verify the expression patterns in Wheat_Chr_Trans_newGene_16173 that were significantly different in the two parents Hongyoumai and Huixianhong. Two RGA4 genes were cloned based on the sequence of Wheat_Chr_Trans_newGene_16173, respectively from two parent and there was one amino acid mutation: $\mathrm{S}$ to $\mathrm{G}$ in Huixianhong on 510 loci.

Conclusion: The combination of SLAF and BSR-seq methods identified a candidate region of pmHYM in the chromosome 7BL of wheat landrace cultivar Hongyoumai. Comparative analysis between the scaffold of co-segregating marker Xmp1207 and SLAF-seq showed five matching blocks. qRT-PCR showed that only the resistant gene Wheat Chr_Trans_newGene_16173 was significantly upregulated in the resistant parent Hongyoumai after inoculation with Bgt, and gene cloning revealed a difference in one amino acid between the two parent genes, indicating it was involved in the resistance response and may be the candidate resistance gene pmHYM.
\end{abstract}

Keywords: Wheat powdery mildew, Resistance gene, Specific-locus amplified fragment sequencing, Bulked segregant analysis, Gene expression

\footnotetext{
*Correspondence: songyuli2000@126.com

${ }^{1}$ Institute of Plant Protection, Henan Academy of Agricultural Sciences; Key Laboratory of Crop Integrated Pest Management of the Southern of North China, Ministry of Agriculture of the People's Republic of China, Zhengzhou 450002, China

Full list of author information is available at the end of the article
}

\section{Background}

Powdery mildew, caused by the obligate biotrophic fungus Blumeria graminis f. sp. tritici (Bgt), is an important disease of wheat [1]. Although wheat powdery mildew can be controlled by fungicides, wheat resistance is the 
safest and most effective means to prevent or slow the spread of the disease. However, co-evolution and the use of single or few Pm genes can lead to rapid development of matching virulence genes in the pathogen population, resulting in breakdown of cultivar resistance $[2,3]$. Therefore, it is important to discover, identify, and clone powdery mildew resistance (PMR) genes.

Mapping and homologous cloning are the major methods used to discover resistance genes; however, gene mapping is the basic of the work. Before the development of high-throughput sequencing, many types of markers, such as RFLP, RAPD, SCAR, STS, CAPs, AFLP, and SSR were used in gene marker identification. This method involved screening primers and fine mapping, which was time-consuming and tedious, thereby limiting studies on candidate genes. Single nucleotide polymorphism (SNP) refers to a single nucleotide mutation that causes DNA sequence diversity at the genome level. SNP markers are becoming major molecular markers, with high distribution density, good genetic stability, and a diallelic genotype, which is easy to detect through high-throughput automated sequencing. The increasing throughput of next-generation sequencing and de novo and referencebased SNP discovery has been applied to several species and is gradually becoming the major method for functional gene mining [4-6]. SLAF-seq, based on automated methods, has a significant advantage of high throughput screening [7]. It is an important method for excavating genomic SNP and a high-resolution strategy for largescale de novo SNP discovery and genotyping. This technology has been made available for haplotype mapping, genetic mapping, linkage mapping, and polymorphism mapping. In previous studies, bulk segregant analysis (BSA), a classical method of marker identification in preliminary screening of primers [8-15], selects 6-10 individuals that represent an extreme phenotype. Due to the small sample sizes, markers identified via this method are usually not closely linked. SLAF uses a minimum of 50 individuals to construct a phenotypic pool, which greatly improves the accuracy of the identified candidate region. Large specific markers of any density that exist throughout the whole genome can be developed based on SLAF-seq to implement fine positioning for functional candidate regions. SLAF-seq has been used for fine mapping and screening of candidate genes in many animals and plants [16-19].

At present, $P m 1 \sim P m 68$ have been named as powdery mildew resistance genes, and $P m 1$ (Pm1a-1e), Pm2 (Pm2a-2c), Pm3 (Pm3a-3j), Pm4 (Pm4a-4d), Pm5 (Pm5a-5e), Pm8 (Pm8 and Pm17), and Pm24 (Pm24a$24 b)$ contain alleles of these resistance genes [20]. However, there has been little progress in whole wheat sequencing because it is difficult to clone genes from polyploid organisms. Until now only a few $P m$ genes have been cloned, including Pm3 [21], Pm38/Yr18/ Lr34/Sr57 [22], Pm8 [23], Pm46/Yr46/Lr67/Sr55 [24], Pm2 [25], Pm60 [26], Pm17 [27], Pm21 [28, 29], Pm24 [30], Pm5e [31], and Pm41 [32].

Of all the identified genes, 30 are derived from common wheat and the remainder are from wild wheat relative species. Exogenous resistance sources were usually used in resistance breeding, sometimes good resistance $(R)$ genes exist in the far-edge materials and are difficult to incorporate. Wheat landraces in China are the products of natural and artificial selection and have better adaptability and important value in the practice of resistance breeding. Previous studies elucidated $P m$ gene composition in some wheat landraces in China and showed that the resistance to wheat powdery mildew in Mazamai, Xiabbaidongmai, Youbailan, Hongjuanmang, Aiganmangmai, Hongtoumai, Dahongtou, Hongyanglazi, Jiantouhong, and Bensanyuehuang were attributable to a single recessive gene [33-37]. These studies enrich our understanding of wheat landraces.

Wheat landrace Hongyoumai was collected and conserved by the Germplasm Resources Bank of the Wheat Research Center at Henan Academy of Agricultural Sciences. This landrace was identified to be highly resistant to wheat powdery mildew at both the seedling and adult stages [38]. Xu constructed a Chancellor"Hongyoumai $\mathrm{F}_{2}$ hybrid cross, and genetic analysis indicated that Hongyoumai carries one dominant resistance gene to powdery mildew [39]. Wang constructed $\mathrm{F}_{2: 3}$ segregating population derived from Yumai13*Hongyoumai and reported that the resistance of Hongyoumai to the Bgt isolate GY was controlled by one dominant resistance gene, temporarily named PmHYM, which was located on chromosome 7BL near SSR markers [40]. Fu constructed $\mathrm{F}_{2: 3}$ segregating population derived from Huixianhong*Hongyoumai, and genetic analysis indicated that the resistance of Hongyoumai to the isolate E09 was controlled by one recessive gene, named pmHYM, and performed fine mapping to identify co-segregating marker Xmp1207 of gene $p m H Y M$ using a $90 \mathrm{k}$ SNP chip [41]. Anti-spectral analysis of 19 wheat powdery mildew pathogen isolates showed that $p m H Y M$ was different from the $P m 5$ gene and its reported allele, and it was a new Pm5 allele or another gene [41]. The virulence frequencies of wheat powdery mildew isolates collected from different areas of Henan for ten years were lower than $40 \%$ according to $\mathrm{Li}$ [42] and our unpublished data, indicating that it can still be used in breeding for Pm resistance. Transcript sequencing was conducted using Yumai13*Hongyoumai $F_{2: 3}$ [43]. However, no candidate resistance genes in Hongyoumai have been identified yet. 
Table 1 Evaluation of sample sequencing data

\begin{tabular}{lllll}
\hline Group & Clean-Reads & Clean-Base & Q30 (\%) & GC (\%) \\
\hline$R_{p}$ & $65,635,954$ & $13,127,190,800$ & 88.62 & 43.41 \\
$S_{p}$ & $62,631,433$ & $12,526,286,600$ & 86.46 & 43.58 \\
F & $30,128,177$ & $6,025,635,400$ & 89.05 & 43.29 \\
M & $35,626,921$ & $7,125,384,200$ & 88.24 & 43.49 \\
\hline
\end{tabular}

In previous research, we have constructed the hybrid combinations of Huixianhong (female parent) $\times$ Hongyoumai (male parent) and done the identification and analysis of the resistance of the susceptible parents Huixianhong, resistant parent Hongyoumai, and $\mathrm{F}_{2: 3}$ individuals dirived from Huixianhong $\times$ Hongyoumai. In this study, we used the SLAF-seq approach to analyze the susceptible parent Huixianhong, resistant parent Hongyoumai, resistant pools $(\mathrm{Rp})$ of $50 \mathrm{~F}_{2: 3}$ homolugous resistant progeny, and susceptible pools (Sp) of $50 \quad \mathrm{~F}_{2}$ susceptible progeny derived from the Huixianhong*Hongyoumai $F_{2: 3}$ population. We identified differentially expressed genes (DEGs) between $\mathrm{Rp}$ of $10 \mathrm{~F}_{2: 3}$ and $\mathrm{Sp}$ of $10 \mathrm{~F}_{2}$ using RNA-seq. Screening and expression patterns of target genes were analyzed in the two parents Hongyoumai and Huixianhong, using gene clone and qRT-PCR, respectively. The aim was to find the candidate region of gene pmHYM and further discover candidate PMR regions in Hongyoumai.

\section{Results}

\section{SNPs accessed using SLAF}

SLAF-seq was conducted using an Illumina HiSeq 2500 at Biomarker Technologies, and $194.02 \mathrm{Mb}$ reads were obtained. Two indicators of sequencing quality, Q30 value and guanine-cytosine (GC) content, were 88.09 and $43.44 \%$, respectively (Table 1 ). Evaluation and monitoring of sequencing data from the control Oryza sativa was used to validate enzymatic digestion in this term, and $388,044,970$ pair-end reads with average comparison efficiency more than $89.08 \%$ using wheat genome as a reference were obtained, along with 614,983 SLAF tags with an average depth of 52.18 in parents and 169.11 in mixed pools (Table 2 and Table 3 ).

A total of 292,753 SNPs were obtained from two parents, containing 851 non-synonymous-coding regions, as well as 392,556 SNPs in two bulks, containing 1283 nonsynonymous-coding regions (Table 4). The distribution of all SNPs on wheat chromosomes is shown in Fig. 1.

A total of 210,495 high-quality SNPs were obtained by filtering out loci with multi-alleles, a read support degree $<4$, genotype consistency in mixed pools and the parent filtered site. Euclidean distance associate analysis was
Table 2 Comparison Statistics with Reference Genomes

\begin{tabular}{llll}
\hline Group & Total-reads & Mapped (\%) & $\begin{array}{l}\text { Properly- } \\
\text { mapped } \\
\text { (\%) }\end{array}$ \\
\hline$R_{p}$ & $13,1271,908$ & 90.15 & 66.88 \\
$S_{p}$ & $125,262,866$ & 89.08 & 65.79 \\
$F$ & $60,256,354$ & 90.71 & 66.50 \\
$M$ & $71,253,842$ & 89.97 & 66.45 \\
\hline
\end{tabular}

Table 3 Statistics of SLAF tags

\begin{tabular}{llll}
\hline Group & SLAF number & Total depth & Average depth \\
\hline$R_{p}$ & 542,168 & $40,619,006$ & 74.9196 \\
$S_{P}$ & 554,172 & $38,178,328$ & 68.8926 \\
$F$ & 367,122 & $18,889,272$ & 51.4523 \\
M & 413,249 & $21,866,603$ & 52.9139 \\
Total & 614,983 & $119,553,209$ & 194.4008 \\
\hline
\end{tabular}

Table 4 SNP annotation result

\begin{tabular}{lll}
\hline Type & F vs $\mathbf{~}$ & $\mathbf{R}_{\mathbf{p}} \mathbf{v s}_{\mathbf{p}}$ \\
\hline INTERGENIC & 211,552 & 282,100 \\
INTRAGENIC & 2 & 1 \\
INTRON & 1919 & 2758 \\
UPSTREAM & 8730 & 11,772 \\
DOWNSTREAM & 7565 & 10,735 \\
UTR_5_PRIMER & 158 & 251 \\
UTR_3_PRIMER & 313 & 468 \\
SPLICE_SITE_ACCEPTOR & 2 & 8 \\
SPLICE_SITE_DONOR & 6 & 15 \\
SPLICE_SITE_REGION & 52 & 86 \\
START_GAINED & 32 & 47 \\
START_LOST & 0 & 2 \\
NON-SYNONYMOUS-START & 1 & 0 \\
SYNONYMOUS-CODING & 1104 & 1455 \\
NON-SYNONYMOUS-CODING & 851 & 1283 \\
STOP-GAINED & 16 & 20 \\
STOP-LOST & 0 & 3 \\
Other & 60,540 & 81,552 \\
Total & 292,753 & 392,556 \\
\hline
\end{tabular}

used to identify a region with a length of $12.95 \mathrm{Mb}$ on chromosome 7BL (Fig. 2 and Table 5).

\section{SNP-index analysis}

SNP-index and $\triangle$ SNP-index distribution of the two bulks is shown in Fig. 3. According to separation ratio theory of progeny in the term, the association threshold was 0.667 . The region above the threshold was screened and 22 regions were 


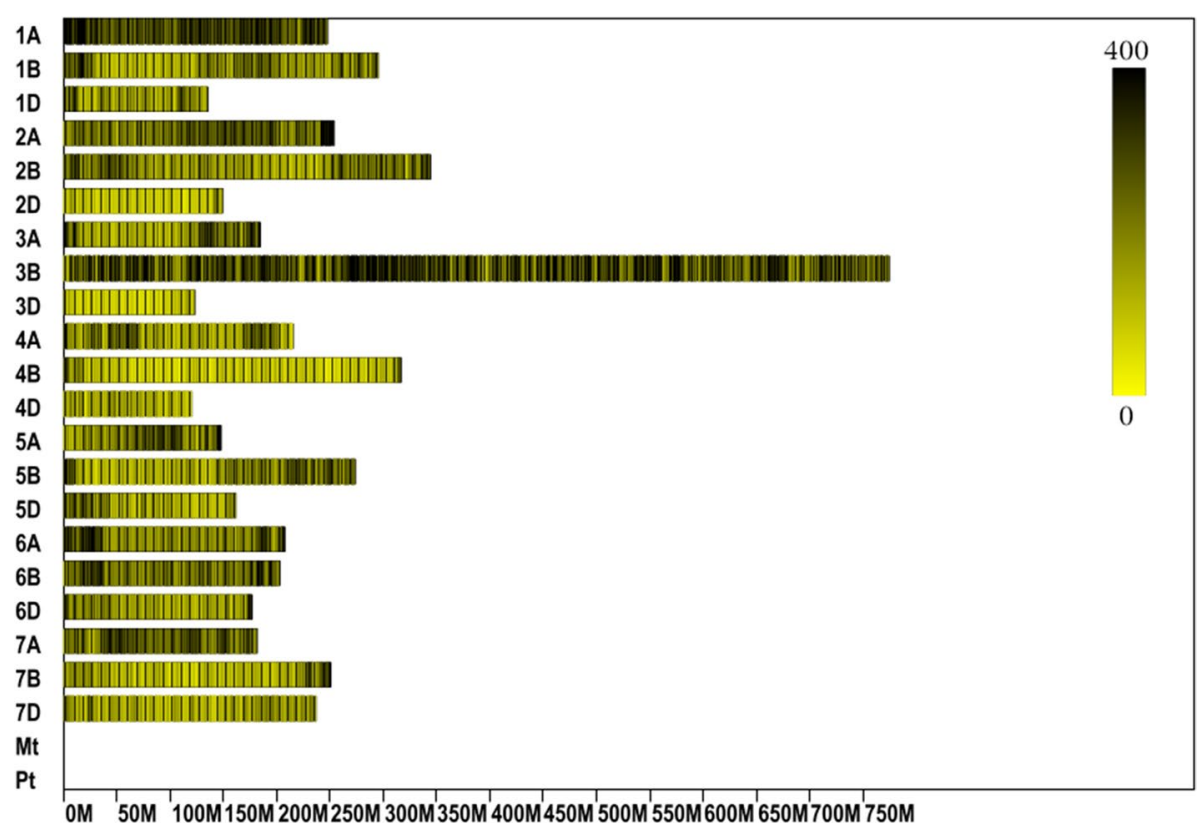

Fig. 1 SNP Distribution on wheat Genome

identified, $40.51 \mathrm{Mb}$ in length and containing 649 genes; of these, one was a non-synonymous-coding gene. The region on chromosome 7BL is shown in Table 6. From the intersection of the Euclidean distance and SNP-index, one candidate region, $12.9547 \mathrm{Mb}$ in length, was identified on 7BL from $237,210,580$ to $250,165,241$, which contained 253 candidate genes. The average distance between the two SLAF markers was $0.0514 \mathrm{cM}$. The region located in the SSR markers range, corresponding to the region of co-segregating marker Xmp1207 derived from TA_TGACv1.30.dna.genome|TGACv1_scaffold_578754_7BL was in five blocks: 237371494-241,751,552; 240,463,691$243,321,483 ; \quad 241,760,892-24,326,939 ; \quad 241,453,352-$ $243,128,561 ; 241,721,380-244,424,206$.

\section{BSR-seq analysis}

\section{Sequence assembly and analysis}

Over 93 million reads were generated, with 51 million reads from T01 (R-pool) and 42 million from T02 (S-pool). A total of $23.62 \mathrm{~Gb}$ clean data were obtained, with a Q30 value of $86.69 \%$ and GC content of $55.96 \%$ (Table 7). The comparison efficiency of reads from two samples T01 and T02 were 70.59 and $71.76 \%$. A total of 11,233 new genes were excavated and 7887 genes were annotated.

\section{Differential expression analysis and candidate gene} analysis

A total of 1311 DEGs were identified, including 1080 upregulated and 231 downregulated with a
Fold-Change $\geq 2.0$ and FDR $<0.01$ criterion. Combining SLAF-BSA and RNA-seq, there were 39 known DEGs, including 11 upregulated and 28 downregulated in the candidate region (Table 8). One gene, transcript Wheat_Chr_Trans_newGene_16173, which codes one putative disease resistance protein RGA4, was identified in co-segregating candidate area $243,334,027-$ $243,335,287$, and $2^{4.67}$-fold upregulated genes were expressed in the R-pool than in the S-pool.

The expression of Wheat_Chr_Trans_newGene_16173 (gene annotation: Putative disease resistance protein RGA4) in Hongyoumai were $2^{3.4}, 2^{3.08}, 2^{3.29}, 2^{2.38}$ at 0,24 , $48,72 \mathrm{~h}$ post inoculation (hpi), respectively, which were higher than those in Huixianhong, indicating that it may be involved in the resistance to wheat powdery mildew (Fig. 4). However, no SNPs or InDels in corresponding regions of gene Wheat_Chr_Trans_newGene_16173 were found in the SLAF results, maybe because the sequences in the corresponding region were not identified by SLAF.

\section{Gene cloning and bioinformation analysis}

Integrated genes $3780 \mathrm{bp}$ in length were obtained in Huixianhong and Hongyoumai blastx analysis (http:// www.ncbi.nlm.nih.gov/), and showed that ORF fragments of Hongyoumai were the same as disease resistance protein RGA4 (Accession no. XM_020324610.1) and one amino acid mutation: $S$ to $G$ in Huixianhong on 510 loci. SMART analysis (http://smart.embl-heide 


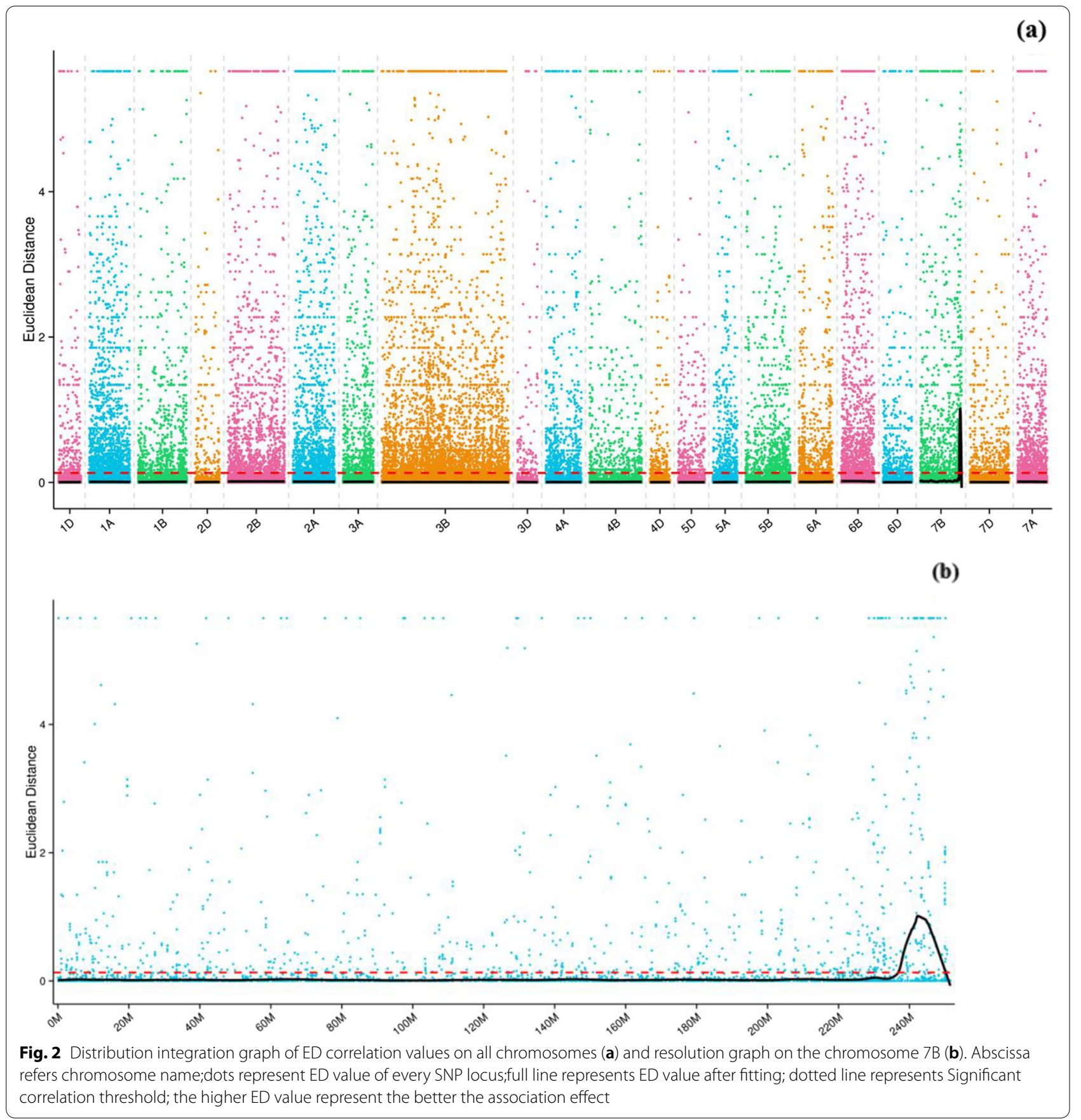

lberg.de/) revealed that coded genes contained seven resistance structure domains: one NB-ARC $(179 \sim 463)$, five leucine-rich repeat (LRR) (597-626; 627-649; 650673 ; 1149-1172; 1173-1196), and one LRR_3 (627940) (Fig. 5).

\section{Discussion}

SLAF is based on SNPs and can identify more abundant polymorphisms than other markers. BSA analysis uses 50 individuals of extreme phenotype. Together they improve
Table 5 Statistical list of associated region information based on ED associate analysis

\begin{tabular}{lllll}
\hline $\begin{array}{l}\text { Chromosome } \\
\text { ID }\end{array}$ & Start & End & Size(Mb) & Gene-Number \\
\hline $7 B$ & $237,210,580$ & $250,165,241$ & 12,9547 & 253 \\
\hline
\end{tabular}


the accuracy of mapping and narrow down the candidate regions. An exact resistance region $12.95 \mathrm{Mb}$ in length on chromosome $7 \mathrm{BL}$ in wheat landrace Hongyoumai was identified in this study using these methods. Further, five blocks were identified by combining the matching scaffold of co-segregating marker Xmp1207 and reducing the scope.

Transcriptome sequencing is an efficient method of screening differential expression transcripts, and is a primary method for obtaining some character-related genes combined with subsequent function analysis. In this study, BSR-seq was used to identify possible candidate genes and identified one candidate resistance transcript Wheat_Chr_Trans_newGene_16173, which was differentially expressed in the two parent types, in the co-segregating region. Blastx results showed that the homologous gene of Wheat_Chr_Trans_newGene_16173 belonged to the RGA4-like resistance gene and integrated ORFs were cloned in Hongyoumai and Huixianhong based on reported disease resistance protein RGA4 [Aegilops tauschii subsp. tauschii] (Accession no. XM_020324610.1).

Resistance genes with LRR domains are widely accepted as playing major roles in plant resistance to pathogens. The LRR domains are thought to interact directly or indirectly with their avirulent effectors to specifically recognize the pathogen. A change in one acid amino in LRR domains may delete or weaken the resistance reaction [44]. Nucleotide-binding site leucine-rich repeat (NBS-LRR) proteins are a current hot topic in plant disease resistance gene research. Genes with NBSLRR domains are an important part of known resistance genes. NBS conserved domains in resistance genes may have specific relationships with pathogen elicitors and downstream proteins of resistance signal transduct. The NBS domain in NBS-LRR proteins is proposed to function as a molecular switch that adjusts the conformation changes of plant resistance proteins through binding ADP or ATP to regulate resistance signal conduction downstream. There are many NBS-LRR resistance genes and many cloned genes of this kind, including Pm3 [21], Pm21 [28, 29], Pm60 [26], Pm5e [31], Pm41 [32], Yr10 [44], Lr1 [45], Lr10 [46], Lr21 [47], Sr33 [48], Sr35 [49], Cre1, and Cre3 [50]. Lr24 [51] and Lr35 [52] are also confirmed NBS genes.

In this study, one wheat powdery mildew resistance gene, a RGA4-like gene, which has one NB-ARC, five
LRR domains, and one LRR_3 was identified and cloned and belonged to the typical NBS-LRR-type gene. Also, one amino acid mutation was observed in the susceptible parent Huixianhong and was differentially expressed in two parents. This suggests that a RGA4-like gene from Hongyoumai may be the candidate $\mathrm{Pm}$ gene or one part plays an important role in resistance to wheat powdery mildew. The amino acid mutation locus was outside the conserved domains, the aa change indicated likely presence of another gene or variation in other regulatory elements, so further study is necessary to discover the mechanism of action of RGA4-like gene in Hongyoumai.

\section{Conclusions}

A candidate region for $p m H Y M, 12.95 \mathrm{Mb}$ in length, was located on chromosome $7 \mathrm{BL}$ in wheat landrace cultivar Hongyoumai using SLAF-BSA. There were five matching blocks between the scaffold of co-segregating marker Xmp1207 and SLAF-seq. Using RNA-seq, 11 upregulated and 28 downregulated transcripts were observed in which a disease resistance protein RGA4 (Wheat_Chr Trans_newGene_16173) was upregulated and expressed in the resistant pool at $16 \mathrm{hpi}$. Further, qRT-PCR results showed Wheat_Chr_Trans_newGene_16173 was aslo obviously higher expression in Hongyoumai (resistant parent) than in Huixianhong (susceptible parent), indicating it maybe play a role in the resistance response.

\section{Methods}

\section{Plant materials and treatments}

All plant materials, including cultivar Huixianhong (female parent) and wheat landrace Hongyoumai (male parent) $F_{2: 3}$ segregating population were derived from Huixianhong $\times$ Hongyoumai. Wheat cultivar Jinfeng 1 code was selected and maintained in the wheat disease research laboratory at the Institute of Plant Protection, Henan Academy of Agricultural Sciences, China. The Bgt E09 race was provided by Prof. Yilin Zhou (Institute of Plant Protection, Chinese Academy of Agricultural Sciences).

Huixianhong, Hongyoumai 50 homozygous resistant and 50 susceptible progeny were planted in nutrition pots and placed in a climate chamber at $16-18^{\circ} \mathrm{C}$, with a light/dark photoperiod of $16 / 8 \mathrm{~h}$, seedlings at the two-leaf and 1 stem stage were collected and mailed to Biomarker Technologies for DNA extraction and SLAF analysis.

(See figure on next page.)

Fig. 3 Distribution integration graph of SNP-index correlation values on all chromosomes (a) and resolution graph on chromosome 7B (b). Abscissa refers chromosome name, color dots represent SNP-index or $\triangle S N P$-index by calculating, black line refers fitting SNP-index or $\triangle S N P$-index. Above figure refers SNP-index value distribution in Rp; middle figure refers SNP-index value distribution in ab pools; bottom figure refers $\triangle S N P$-index value distribution and red line refers theoretical correlation threshold line 


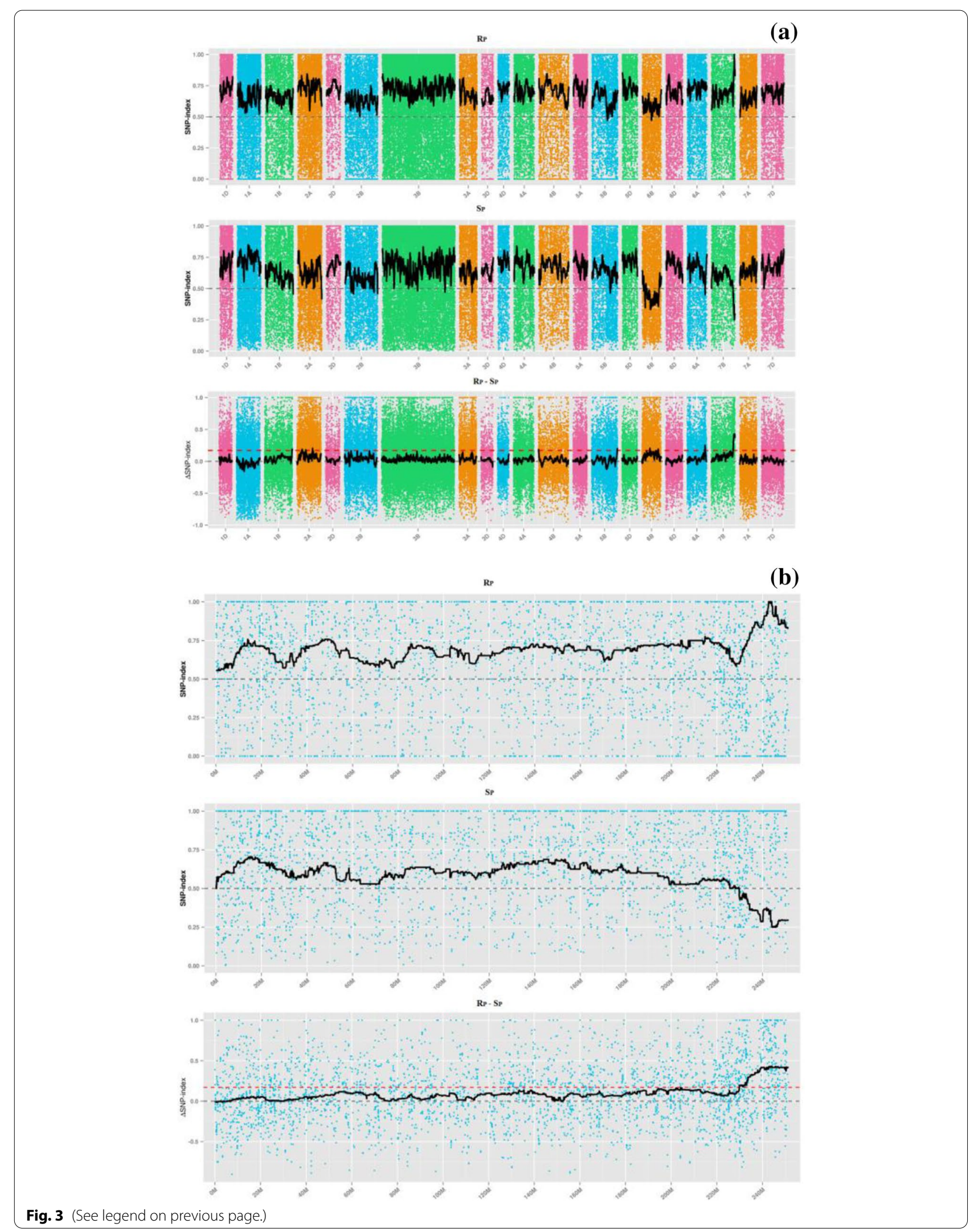


Table 6 Statistical list of associated region information based on SNP-index

\begin{tabular}{lllll}
\hline $\begin{array}{l}\text { Chromosome } \\
\text { ID }\end{array}$ & Start & End & Size(Mb) & Gene-Number \\
\hline $7 B$ & $229,913,510$ & $251,503,216$ & 21.59 & 382 \\
\hline
\end{tabular}

Table 7 Evaluation of sample sequencing data by RNA-seq

\begin{tabular}{llllll}
\hline Sample & BMK-ID & Clean reads & Clean bases & GC Content & $\% \geq \mathbf{Q 3 0}$ \\
\hline R-pool & T01 & $51,493,576$ & $12,962,382,281$ & $56.62 \%$ & $86.61 \%$ \\
S-pool & T02 & $42,324,424$ & $10,657,325,621$ & $55.30 \%$ & $86.77 \%$ \\
\hline
\end{tabular}

Two bulk DNA of the $\mathrm{F}_{2: 3}$ segregating population were developed using Huixianhong as susceptible and Hongyoumai as resistance donors. BSA was conducted on 50 resistant and 50 susceptible progeny.

Ten susceptible progeny and ten $\mathrm{F}_{2: 3}$ resistant progeny were planted in nutrition pots and maintained in a growth chamber at $16-18^{\circ} \mathrm{C}$, with a light/dark photoperiod of 16/8h. Inoculation with the Bgt E09 race (maintained on susceptible wheat cultivar Jinfeng 1 code) was performed by shaking conidia onto wheat leaves when plants were at the three-leaf stage. At $16 \mathrm{~h}$ post inoculation (hpi), leaves were individually collected, wrapped in tin foil, frozen in liquid nitrogen, and immediately stored at $-80^{\circ} \mathrm{C}$. Then, samples were sent in dry ice to Biomaker Technologies for RNA-sequencing.

Hongyoumai and Huixianhong were planted and inoculated with the Bgt E09 race. The inoculated and control wheat leaves were collected at $0,24,48$, and $72 \mathrm{hpi}$, and were immediately frozen in liquid nitrogen and stored at $-80^{\circ} \mathrm{C}$ prior to extraction of total RNA.

\section{SLAF library construction and sequencing}

Total genomic DNA was extracted from wheat leaves according to the cetyltrimethylammonium bromide (CTAB) method. DNA quality and concentration were estimated using an ND-1000 spectrophotometer (Nanodrop, Wilmington, DE, USA) and electrophoresis in $0.8 \%$ agarose gels with a lambda DNA marker. Wheat genome sequences were constructed as a reference to calculate electronic enzyme digestion using restriction enzymes Rsal. The SLAF library was constructed as described by Sun et al [7], with steps as follows: Genomic DNA of each sample was digested using RsaI and a single nucleotide (A) overhang was added to the digested fragments. Dual-index sequencing adapters were ligated to the A-tailed fragments using $\mathrm{T}_{4}$ ligase. Then, the fragments were amplified using PCR and the products were separated using 2.0\% agar electrophoresis. Fragments of
364-394 bp in length were excised, purified, and sequenced to obtain target fragments. Pair-end sequencing was conducted using an Illumina HiSeq 2500 system (Illumina, Inc., San Diego, CA, USA) according to the manufacturer's recommendations (Beijing Biomarker Company).

\section{SLAF-seq data analysis}

SLAF-seq identification and genotyping were performed as described by Sun [7] and Zhang [53]. To assure the quality of sequence analysis, two read lengths of $100 \mathrm{bp}$ were used for data evaluation and analysis. The first $100 \mathrm{bp}$ and last $100 \mathrm{bp}$ in one sequence were both evaluated with a quality score Q30 (indicating a $0.1 \%$ chance of error, and 99.9\% confidence). Reads $<\mathrm{Q} 30$ were filtered out and the ratio of the number of high-quality reads with quality scores $>$ Q30 (indicating a $0.10 \%$ chance of error and $99.90 \%$ confidence) were added to the total number of raw reads and the GC content was calculated. After the barcodes and the terminal 5 bp positions were trimmed, the high-quality reads were mapped onto the reference genome sequence using SOAP software [54]. Sequences mapping to the same position with over $95 \%$ identity were grodowned into one SLAF locus [53]. The sequence error rate was estimated using the Oryza sativa genome sequence data as the control. SLAF-tags were identified in parents and mixed pools, and the SLAF-tags that were polymorphic in the female (Hongyoumai) and male (Huixianhong) parents were considered as SLAF markers for subsequent analysis.

\section{SNP accession and SNP-index analysis}

SNP is the major form of polymorphic SLAF. SNP detection was conducted using GATK software and SAM tools [55]. SNPs with common variant loci identified by the two methods were considered valid. SnpEff $[55,56]$ was used to note and forecast the resulting variation (SNP, Small InDel). Variation regions and effects were identified according to the location and gene information of various loci on the reference genome. High-quality SNPs were obtained as follows. First, SNP loci with many genotypes and reads with a downport degree $<4$ bp were filtered out. Second, SNP loci that were coincident between Rp and Sp and genes of Rp did not come from the resistant parent were also filtered out. Third, credible SNPs were obtained. Association analysis was conducted using two methods - Euclidean distance and SNPindex $[55,57,58]$. A candidate region was identified from the intersection of the ED and SNP-index analysis. Genes in the candidate region were annotated using BLAST and the databases NR, Swiss-Prot, GO, KEGG, and COG [57-60].

The $\triangle \mathrm{SNP}$-index was calculated using significant differences between bulk genotype frequency. $\Delta$ (SNP-index values approaching 1 indicated more SNP markers linked to the target trait. 
Table 8 Differential expressed genes in candidate region

\begin{tabular}{|c|c|c|c|}
\hline Genes \#ID & $\log _{2} \mathrm{FC}$ & regulated & nr_annotation \\
\hline Wheat_Chr_Trans_newGene_16167 & 6.745021314 & up & $\begin{array}{l}\text { PREDICTED: polyadenylate-binding protein-interacting protein 8-like [Brachy- } \\
\text { podium distachyon] }\end{array}$ \\
\hline Wheat_Chr_Trans_newGene_16173 & 4.657390482 & up & Putative disease resistance protein RGA4 [Aegilops tauschii] \\
\hline Wheat_Chr_Trans_newGene_16408 & 3.857153144 & up & PREDICTED: putative F-box protein At4g17565-like [Setaria italica] \\
\hline gene:Traes_7BL_E41865FE0 & 2.431156696 & up & Putative coatomer subunit beta'-3 [Aegilops tauschii] \\
\hline gene:Traes_7BL_97AC3748A & 1.914289176 & up & predicted protein [Hordeum vulgare subsp. vulgare] \\
\hline gene:Traes_7BL_418261D09 & 1.844998764 & up & hypothetical protein ZEAMMB73_490100 [Zea mays] \\
\hline gene:Traes_7BL_4437E5002 & 1.799818185 & up & E3 ubiquitin-protein ligase KEG [Triticum urartu] \\
\hline gene:Traes_7BL_A54AA7230 & 1.594932596 & up & hypothetical protein F775_06825 [Aegilops tauschii] \\
\hline gene:Traes_7BL_C09CECD07 & 1.31525402 & up & Transcriptional repressor NF-X1 [Aegilops tauschii] \\
\hline gene:Traes_7BL_E8D5F3A5E & 1.159372618 & up & predicted protein [Hordeum vulgare subsp. vulgare] \\
\hline gene:Traes_7BL_FD4254327 & 1.102683172 & up & vacuolar proton-inorganic pyrophosphatase [Hordeum vulgare subsp. vulgare] \\
\hline Wheat_Chr_Trans_newGene_16401 & -5.91836062 & down & - \\
\hline Wheat_Chr_Trans_newGene_16402 & -5.888805499 & down & - \\
\hline Wheat_Chr_Trans_newGene_16164 & -5.731202485 & down & T-complex protein 1 subunit beta [Triticum urartu] \\
\hline gene:Traes_7BL_95D9D023E & -4.83760605 & down & speckle-type POZ protein [Zea mays] \\
\hline gene:Traes_7BL_8B348AE18 & -4.746575637 & down & predicted protein [Hordeum vulgare subsp. vulgare] \\
\hline gene:Traes_7BL_EA8DE756D & -4.737738698 & down & Putative flavin-containing monooxygenase 1 [Aegilops tauschi] \\
\hline Wheat_Chr_Trans_newGene_16411 & -4.609251169 & down & predicted protein [Hordeum vulgare subsp. vulgare] \\
\hline Wheat_Chr_Trans_newGene_16397 & -4.566074614 & down & T-complex protein 1 subunit beta [Triticum urartu] \\
\hline Wheat_Chr_Trans_newGene_16399 & -4.13389143 & down & Speckle-type POZ protein-like protein B [Aegilops tauschii] \\
\hline gene:Traes_7BL_466F701B8 & -4.106259873 & down & predicted protein [Hordeum vulgare subsp. vulgare] \\
\hline gene:Traes_7BL_AF173EC17 & -4.090072315 & down & hypothetical protein TRIUR3_06034 [Triticum urartu] \\
\hline gene:Traes_7BL_F45C15FA2 & -4.048001723 & down & RecName: Full=HMG1/2-like protein [Triticum aestivum] \\
\hline Wheat_Chr_Trans_newGene_16409 & -3.873600139 & down & hypothetical protein F775_24416 [Aegilops tauschii] \\
\hline gene:Traes_7BL_OFBOAC067 & -3.344018347 & down & hypothetical protein F775_07814 [Aegilops tauschii] \\
\hline gene:Traes_7BL_AEE3C83EC & -3.090935295 & down & predicted protein [Hordeum vulgare subsp. vulgare] \\
\hline gene:Traes_7BL_DE9710474 & -3.012246392 & down & predicted protein [Hordeum vulgare subsp. vulgare] \\
\hline gene:Traes_7BL_BAFAEOAE2 & -2.97032921 & down & Protease 2 [Aegilops tauschii] \\
\hline gene:Traes_7BL_B97BBAFAD & -2.867453779 & down & PREDICTED: callose synthase 3-like [Brachypodium distachyon] \\
\hline Wheat_Chr_Trans_newGene_16174 & -2.647997075 & down & - \\
\hline gene:Traes_7BL_FA7046752 & -2.592244631 & down & hypothetical protein F775_24157 [Aegilops tauschii] \\
\hline Wheat_Chr_Trans_newGene_16410 & -2.565235722 & down & Putative disease resistance protein RGA3 [Aegilops tauschii] \\
\hline gene:Traes_7BL_70E7494E5 & -2.432573595 & down & ent-copalyl diphosphate synthase [Triticum aestivum] \\
\hline gene:Traes_7BL_8A1D0A241 & -2.291330273 & down & Ent-copalyl diphosphate synthase 1, chloroplastic [Triticum urartu] \\
\hline gene:Traes_7BL_7A3B8A199 & -1.975271473 & down & RecName: Full= Catalase isozyme 1 [Hordeum vulgare] \\
\hline gene:Traes_7BL_FB212B9AE & -1.960701644 & down & oligopeptidase B [Triticum aestivum] \\
\hline gene:Traes_7BL_59EFF59F1 & -1.937327117 & down & predicted protein [Hordeum vulgare subsp. vulgare] \\
\hline Wheat_Chr_Trans_newGene_16407 & -1.92007674 & down & - \\
\hline gene:Traes_7BL_ACE459781 & -1.712545481 & down & predicted protein [Hordeum vulgare subsp. vulgare] \\
\hline
\end{tabular}

$$
\begin{aligned}
& \text { SNP_index }(R p)=M R p /(P R p+M R p) \\
& \text { SNP_index }(\mathrm{Sp})=M S p /(P S p+M S p) \\
& \Delta\left(S N P \_ \text {index }\right)=\text { SNP_index }(R p)-S N P \_ \text {index }(S p)
\end{aligned}
$$

where MRp indicates the depth of $\mathrm{Rp}$ in the male parent, PRp indicates the depth of $\mathrm{Rp}$ in the female parent,
MSp indicates the depth of Sp in the male parent, and PSp indicates the depth of Sp in the female parent. False positive loci were filtered out by fitting the $\triangle \mathrm{SNP}$-index value of SNP markers from the same chromosome.

\section{BSR-seq analysis}

RNA-seq was conducted using Illumina HiSeq sequencing with two pooled samples of resistance $\mathrm{F}_{2: 3}$ and susceptible 


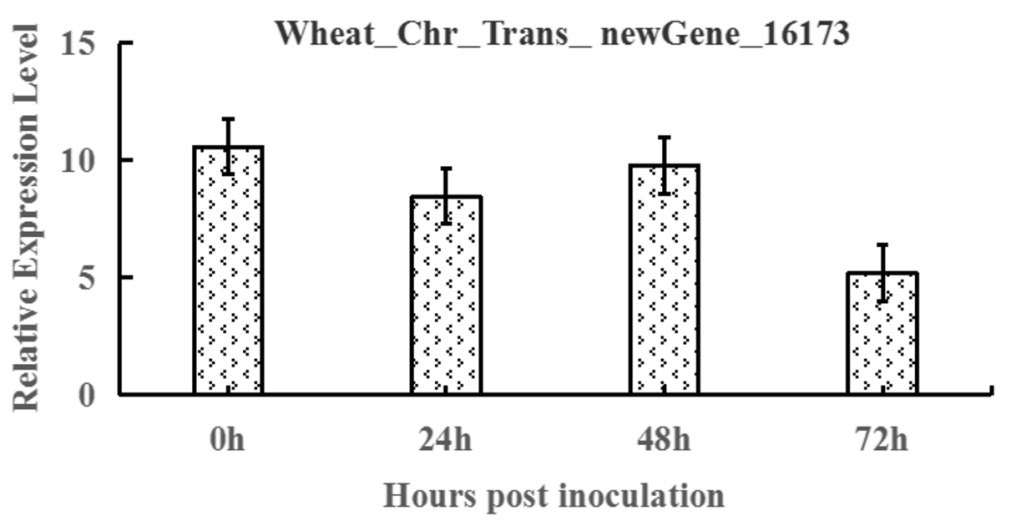

Fig. 4 Expression analysis of Wheat_Chr_Trans_newGene_16173. Relative expression level of Wheat_Chr_Trans_newGene_16173 in parent Hongyoumai than Huixianhong at the same time point after inoculation

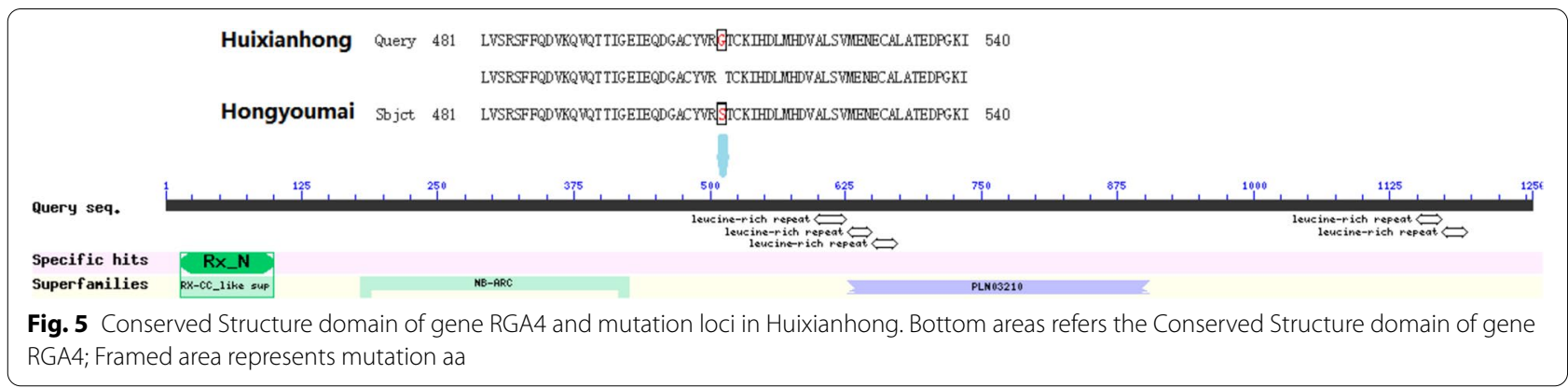

offspring. Each pooled sample contained equal amounts of RNA collected from 10 individuals. Ten resistant plants and 10 susceptible plants were used individually for RNA extracts following the instruction manual of the Trizol Reagent (Life technologies, California, USA). RNA integrity and concentration were checked using a Nanodrop ND-1000 spectrophotometer (Thermo Scientific, Wilmington, DE, USA) and Agilent 2100 Bioanalyzer (Agilent Technologies, Santa Clara, CA, USA). Equal amounts of RNA of qualified samples constituted T01 (R-pool) and T02 (S-pool) for library construction as follows. First, mRNA was isolated using NEBNext Poly (A) mRNA Magnetic Isolation Module (NEB, E7490) and the enriched mRNA was fragmented into approximately $200 \mathrm{nt}$ RNA inserts, which were used to synthesize the first-strand cDNA and the second cDNA. The double-stranded cDNA was endrepaired, de-A-tailed and adaptor ligated. The suitable fragments were isolated using Agencourt AMPure XP beads (Beckman Coulter, Inc.), and enriched using PCR to obtain the cDNA library. Qubit 2.0 and Agilent 2100 were used to detect the concentration and insert size of the cDNA library, respectively, and qPCR was used to detection the exact quantity for effective concentration of the library. Finally, the qualified cDNA libraries were sequenced using an Illumina HiSeq 2500 sequencing platform.
Reads containing adapter sequences, unknown nucleotides $>5 \%$, and low-quality reads of the raw sequencing data, were removed to obtain high-quality clean data $(\mathrm{Q} 30>85 \%)$. Then, clean reads were mapped to the wheat genome (ftp://ftp.ensemblgenomes.org/pub/ plants/release-30/fasta/triticum_aestivum/dna/) using TopHat2. Mapping data, alternative splicing analysis, gene-structure optimization analysis, and the discovery of new genes were performed and gene expression levels were calculated using the reads per kilobase per million mapped reads (RPKM) method. Clean Reads from two samples were used for comparative analysis with the reference genome using TopHat2 Transcripts were filtered for a fold-change threshold of $\geq 2.0$ and $\leq 0.5$. The False Discovery Rate (FDR) $<0.01$ was applied to identify significantly differentially expressed genes.

\section{qRT-PCR verification}

Total RNA was isolated from wheat leaf extracts using Trizol reagent (TAKARA Biomedical Technology) according to the manufacturer's instructions. The first strand of cDNA was synthesized from $2 \mu \mathrm{g}$ of the total RNA using RT-PCR (TAKARA). The cDNA samples were diluted $\times 10$ and used as the templates for 
qRT-PCR, which was performed on an ABI PRISM 7500 Real-Time PCR System (Applied Biosystems, Foster City, CA, USA). According to the sequence of the candidate gene Wheat_Chr_Trans_newGene_16173 (Putative disease resistance protein RGA4), forwards primer GGGGTCCTCCATCTTC and reverse primer TGGTGCCCAGCCGTT were synthesized to analyze the transcript levels in Hongyoumai and Huixianhong. Wheat 26S rRNA (GenBank accession no. Z11889.1) was used as a control normalization parameter, with primers as follows: 26S-F: GAAGAAGGTCCCAAG GGTTC; 26S-R: TCTCCCTTTAACACCAACGG. The reaction system was as follows: $2 \times$ qPCR Mix $10 \mu \mathrm{L}$, $2 \mu \mathrm{L}$ cDNA, and $1 \mu \mathrm{L} / 10 \mu \mathrm{mol} \mathrm{L}^{-1}$ of each primer, with $\mathrm{H}_{2} \mathrm{O}$ to a total volume of $20 \mu \mathrm{L}$. The temperature cycle program was $95^{\circ} \mathrm{C}$ for $10 \mathrm{~min}, 95^{\circ} \mathrm{C}$ for $10 \mathrm{~s}, 55^{\circ} \mathrm{C}$ for $30 \mathrm{~s}$, and $72^{\circ} \mathrm{C}$ for $34 \mathrm{~s}$ for 40 cycles. All reactions were performed in triplicate, including three non-template controls. Dissociation curves were generated for each reaction to ensure specific amplification. Transcript concentration was calculated using $2^{-\triangle \triangle \mathrm{Ct}}$ [61].

\section{Gene cloning}

Primers were synthesized based on the ORF of the sequence of putative disease resistance protein RGA4 [Aegilops tauschii subsp. tauschii] (Accession no. XM_020324610.1) to amplify full length gene sequences of Hongyoumai and Huixianhong. Forwards primer: 5840-F: ATGGCGGCGACGGT; reverse primer: 5840R:CTAATCTCTACGGATGGCACATTTCC. Reaction volume: $2 \times$ fast pfu master mix $25 \mu \mathrm{L}, 1 \mu \mathrm{L} 5840-\mathrm{F}$, $1 \mu \mathrm{L} 5840-\mathrm{R}, 3 \mu \mathrm{L}$ cDNA, with $\mathrm{H}_{2} \mathrm{O}$ up to $50 \mu \mathrm{L}$ reaction volume. The PCR amplification cycle was $94{ }^{\circ} \mathrm{C}$ for $3 \mathrm{~min}, 94{ }^{\circ} \mathrm{C}$ for $30 \mathrm{~s}, 58{ }^{\circ} \mathrm{C}$ for $1 \mathrm{~min}, 72{ }^{\circ} \mathrm{C}$ for $1 \mathrm{~kb} / 30 \mathrm{~s}$ for 33 cycles, followed by $72{ }^{\circ} \mathrm{C}$ for $10 \mathrm{~min}$. Target gene fragments were isolated using $1.0 \%$ agarose gel electrophoresis recovery, purification, and sequencing.

\begin{abstract}
Abbreviations
Bgt: Blumeria graminis f. sp. tritici; NGS: Next-Generation Sequencing; SLAF: Specific-Locus Amplified Fragment Sequencing; BSA: Bulked segregant analysis; hpi: hours post inoculation; DEGs: Differential expressed genes; qRT-PCR: Quantitative reverse transcription-Polymerase Chain Reaction; aa: amino acid; PMR: powdery mildew resistance; SNP: Single nucleotide polymorphism,; NBSLRR: Nucleotide binding site-leucine-rich repeats; GC: guanine-cytosine; ED: Euclidean Distance; RPKM: the reads per kilobase per million mapped reads; FDR: False Discovery Rate.
\end{abstract}

\section{Acknowledgements}

Thank Professor Zhou Yilin (Institute of Plant Protection, Chinese Academy of Agricultural Sciences) kindly provides us with Bgt E09.

Ethical statement for experimental research and field studies on plants Experimental research and field studies on plants complies with relevant institutional, national, and international guidelines and legislation.

\section{Authors' contributions}

WJ and SY were responsible for the data analysis and experimental design. WJ wrote the manuscript. WJ, LY, XF, XH, HZ, LL and SY were responsible for the programs and the manuscript revision. All authors have commented, read and approved the final manuscript.

\section{Funding}

This study was financially supported by the National Key Research and Development Project (grant no.

2016YFD0300705) and Henan Wheat Industry Technology System (no. S2010-01-G05).

\section{Declarations}

Ethics approval and consent to participate

Not applicable.

\section{Consent for publication}

Not applicable.

\section{Competing interests}

The authors declare that they have no competing interests.

\section{Author details}

${ }^{1}$ Institute of Plant Protection, Henan Academy of Agricultural Sciences; Key Laboratory of Crop Integrated Pest Management of the Southern of North China, Ministry of Agriculture of the People's Republic of China, Zhengzhou 450002, China. ${ }^{2}$ School of Life Sciences, Henan University, Kaifeng 475001, China.

Received: 19 May 2021 Accepted: 27 January 2022

Published online: 23 February 2022

References

1. Xie H, Chen X, Sheng BQ, Xin ZY, Kong FJ, Lin ZS, et al. Identification of a wheat line $Y W 243$ resistance to powdery mildew and genetic analysis. Acta Agron Sin. 2001;27-6:715-21.

2. Li H, Chen X, Xin ZY, Ma YZ, Xu HJ. Development and identification of wheat-Haynaldia villosa 6DL/6VS translocation lines with powdery mildew resistance (in Chinese). Sci Agric Sin. 1999;32(5):9-15.

3. Liu JY, Liu DJ, Tao WJ, Li WL, Chen PD. Study on the conversion of RFLP markers co-segregated with Pm4a to sequenced-tagged-site markers. J Agri Biotech. 1999;7(2):113-6.

4. Schneeberger K, Weigel D. Fast-forward genetics enabled by new sequencing technologies. Trends Plant Sci. 2011;16:282-8.

5. Shi W, Eli M, John KM, Mikhail VM. 2b-RAD:a simple and flexible method for genome-wide genotyping. Nat Methods. 2012;9:808-10.

6. Mokry M, Nijman IJ, van Dijken A, Benjamins R, Heidstra R, Scheres B, et al. Identification of factors required for meristem function in Arabidopsis using a novel next generation sequencing fast forward genetics approach. BMC Genomics. 2011;12:256.

7. Sun XW, Liu DY, Zhang XF, Li WB, Liu H, Hong WG, et al. SLAF-seq: an efficient method of large-scale De novo SNP discovery and genotyping using high-throughput sequencing. PLoS One. 2013;8:e57900.

8. Ruyter-Spira CP, Gu Z, Van der PJ, Groenen M. Bulked segregant analysis using microsatellites: mapping of the dominant white locus in the chicken. Poult Sci. 1997;76:386-91.

9. Becker A, Chao DY, Zhang X, Salt DE, Baxter I. Bulk segregant analysis using single nucleotide polymorphism microarrays. PLoS One. 2011;6:e15993.

10. Suo GL, Huang ZJ, He CF, Shen YZ, Wang J. Identification of the molecular markers linked to the salt-resistance locus in the wheat using RAPD-BSA technique. Acta Bot Sin. 2001:43:598.

11. Dussle C, Quint M, Melchinger A, Xu M, Lübberstedt T. Saturation of two chromosome regions conferring resistance to SCMV with SSR and AFLP markers by targeted BSA. Theor Appl Genet. 2003;106:485-93.

12. Lee BY, Penman D, Kocher T. Identification of a sex-determining region in Nile tilapia (Oreochromis niloticus) using bulked segregant analysis. Anim Genet. 2003;34:379-83. 
13. Asnaghi C, Roques D, Ruffel S, Kaye C, Hoarau JY, Telismart H, et al. Targeted mapping of a sugarcane rust resistance gene (Bru1) using bulked segregant analysis and AFLP markers. Theor Appl Genet. 2004;108:759-64.

14. Cheema KK, Grewal NK, Vikal Y, Sharma R, Lore JS, Das A, et al. A novel bacterial blight resistance gene from Oryza nivara mapped to $38 \mathrm{~kb}$ region on chromosome $4 \mathrm{~L}$ and transferred to Oryza sativa L. Genet Res. 2008;90:397.

15. Zhao SH, Zhu MJ, Chen HB. Immunogenomics for identification of disease resistance genes in pigs: a review focusing on gram-negative bacilli. J Anim Sci. 2012;3(34):1-13.

16. Chen S, Huang Z, Dai Y, Qin S, Gao Y, Zhang L, et al. The development of 7E chromosome-specific molecular markers for Thinopyrumelongatum based on SLAF-seq technology. PLoS One. 2013;8:e65122.

17. Zhang Y, Wang L, Xin H, Li D, Ma C, Ding X, et al. Construction of a highdensity genetic map for sesame based on large scale marker development by specific length amplified fragment (SLAF) sequencing. BMC Plant Biol. 2013;13:141.

18. Hu MJ, Zhang HP, Liu K, Cao JJ, Wang SX, Jiang H, et al. Cloning and characterization of TaTGW-7A gene associated with grain weight in wheat via SLAF-seq-BSA. Front Plant Sci. 2016;7:1902. https://doi.org/10.3389/fpls.

19. Yin JL, Fang ZW, Sun C, Zhang P, Zhang X, Lu C, et al. Rapid identification of a stripe rust resistant gene in a space-induced wheat mutant using specific locus amplified fragment (SLAF) sequencing. Sci Rep-UK. 2018;8:3086.

20. McIntosh RA, Dubcovsky J, Rogers WJ, Morris C, Xia XC. Catalogue of gene symbols for wheat: 2017 supplement (KOMUGI Wheat Genetic Resource Database). https://shigen.nig.ac.jp/wheat/komugi/genes/symbolClassList.jsp.

21. Yahiaoui N, Srichumpa P, Dudler R, Keller B. Genome analysis at different ploidy levels allows cloning of the powdery mildew resistance gene Pm3b from hexaploid wheat. J Mol Cell Biol. 2004;37:528-38.

22. Krattinger SG, Lagudah ES, Spielmeyer W, Singh RP, Huerta-Espino J, McFadden $\mathrm{H}$, et al. A pu-tative $\mathrm{ABC}$ transporter confers durable resistance to multiplefungal pathogens in wheat. Science. 2009:323:1360-3.

23. Hurni S, Brunner S, Buchmann G, Herren G, Jordan T, Krukowski P, et al. Rye $P m 8$ and wheat $P m 3$ are orthologous genes and show evolutionary conservation of resistance function against powdery mildew. Plant J. 2013;76:957-69.

24. Moore JW, Herrera-Foessel S, Lan C, Schnippenkoetter W, Ayliffe M, HuertaEspino J, et al. A recently evolved hexose transporter variant confers resistance to multiple pathogens in wheat. Nat Genet. 2015;47:1494-8.

25. Sanchez-Martin J, Steuernagel B, Ghosh S, Herren G, Hurni S, Adamski $\mathrm{N}$, et al. Rapid gene isolation in barley and wheat by mutant chromosome sequencing. Genome Biol. 2016;17:221.

26. Zou SH, Wang H, Li YW, Kong ZS, Tang DZ. The NB-LRR gene Pm60 confers powdery mildew resistance in wheat. New Phytol. 2017;218:298-309.

27. Singh SP, Hurni S, Ruinelli M, Brunner S, Sanchez-Martin J, Krukowski P, et al. Evolutionary divergence of the rye $P m 17$ and $P m 8$ resistance genes reveals ancient diversity. Plant Mol Biol. 2018;98:249-60.

28. Xing LP, Hu P, Liu JQ, Witek K, Zhou S, Xu JF. Pm21 from Haynaldiavillosa encodes a CC-NBS-LRR protein conferring powdery mildew resistance in wheat. Mol Plant. 2018;11:874-8.

29. He HG, Zhu SY, Zhao RH, Jiang ZN, Ji YY, Ji J, et al. Pm21, encoding a typical CC NBS-LRR protein, confers broad-spectrum resistance to wheat powdery mildew disease. Mol Plant. 2018;11:879-82.

30. Lu P, Guo L, Wang ZZ, Li BB, Li J, Li YH, et al. A rare gain of function mutation in a wheat tandem kinase confers resistance to powdery mildew. Nat Commun. 2020;11:680

31. Xie JZ, Guo GH, Wang Y, Hu TZ, Wang LL, Li JT, et al. A rare single nucletide variant in Pm5e confers powdery mildew resistance in common wheat. New Phytol. 2020;228(3):1011-26.

32. Li MM, Dong LL, Li BB, Wang ZZ, Xie JZ, Qiu D, et al. A CNL protein in wild emmer wheat confers powdery mildew resistance. New Phytol. 2020;228(3):1027-37.

33. Xiong EH, Zhu W, Cao Y, Chai SB, Fang XW. A genetic analysis of powdery mildew resistance in three native wheat varieties. J Jiangsu Agri College. 1995;16(2):47-50.

34. Xue F, Duan XY, Zhou YL, Ji WQ. Postulation of powdery mildew resistant genes carried in some chinese wheat landraces and the genetic diversity analysis. J Triticeae Crops. 2009;29:228-35.
35. Xiao MG. Identification of the genes conferring resistance to powdery mildew in the Chinese landraces. PhD thesis, Chinese Academy of Agricultural Sciences. Institute of Plant Protection; 2013.

36. Xu HX, Xu YF, Geng LG, An DG. Resistance of Chinese wheat landraces and relatives at seedling stage to powdery mildew. Chin J Eco-Agric. 2011;19:1210-4

37. Xu XD. Identification and mapping of powdery mildew resistance genes from 5 wheat landraces. PhD thesis. China Agricultural University. College of Plant Protection; 2017.

38. Wang XF, Zhang ZS, Liu HY, He WL. Evaluation of resistance and slowmildewing of some wheat varieties onHenan province. Acta Agriculture Universitatis Henanensis. 1996;30:160-3.

39. Xu HX. Development of molecular markers linked to five genes for the powdery mildew resistance in wheat. MS thesis. Henan University. School of life sciences; 2004

40. Wang JM, Liu HY, Kang ZS. Genetic analysis and location of a powdery mildew resistance gene in wheat landrace Hongyoumai by SSR marker. Acta Phytopathologica Sinica. 2009;39:285-9.

41. Fu BS, Zhang ZL, Zhang QF, Wu XY, Wu JZ, Cai SB. Identification and mapping of a new powdery mildew resistance allele in the Chinese wheat landrace Hongyoumai. Mol Breed. 2017;37:133.

42. Li YH, Wang JM, Xu F, Yang GQ, Song YL, Liu LL. Virulence structure of the wheat powdery mildew population in Henan Province in China during 2011 2014. Acta Phytopathologica Sinica. 2016;4:573-6.

43. Wang JM, Liu HY, Xu HM, Li M, Kang ZS. Analysis of differential transcriptional profiling in wheat infected by Blumeria graminis f. sp. tritici using genechip. Mol Biol Rep. 2012;39:381-7.

44. Liu W, Frick M, Huel R, Nykiforuk CL, Wang XM, Gaudet DA, et al. The stripe rust resistance gene Yr10 encodes an evolutionary-conserved and unique CC-NBS-LRR sequence in wheat. Mol Plant. 2014;7:1740-55.

45. Cloutier S, Mccallum BD, Loutre C, BanksTWWT, Feuillet $C$, et al. Leaf rust resistance gene $L r 1$, isolated from bread wheat (Triticum aestivum $L$.) is a member of the large psr567 gene family. Plant Mol Biol. 2007;65:93-106.

46. Sela H, Spiridon LN, Petrescu AJ, Akerman M, Mandel-Gutfreund Y, Nevo E, et al. Ancient diversity of splicing motifs and protein surfaces in the wild emmer wheat (Triticum dicoccoides) LR10 coiled coil (CC) and leucine-rich repeat (LRR) domains. Mol Plant Pathol. 2012;13:276-87.

47. Huang L, Brooks SA, Li W, Fellers JP, Trick HN, Gill BS. Map-based cloning of leaf rust resistance gene $L r 21$ from the large and polyploid genome of bread wheat. Genet. 2003:164:655-64.

48. Periyannan S, Moore J, Ayliffe M, Clemence M, Zhang JP, Zhang P, et al. The gene Sr33, an ortholog of barley Mla genes, encodes resistance to wheat stem rust race Ug99. Sci. 2013;341:786-8.

49. Saintenac C, Zhang W, Salcedo A, Rouse MN, Trick HN, Akhunov E, et al. Identification of wheat gene Sr35 that confers resistance to Ug99 stem rust race group. Sci. 2013;341:783-6.

50. De Majnik J, Ogbonnaya FC, Moullet O, Lagudah E. The cre1 and cre3 nematode resistance genes are located at homeologous loci in the wheat genome. Mol Plant Microbe In. 2003;16:1129-34.

51. Zhang LR, Yang WX, Liu DQ. Diversity and evolutionary relationship of NBStype resistance gene analogues in wheat. Acta Agriculturae Boreali Sinica. 2011;26(4):23-6.

52. Gao Q, Wang HY, Liu DQ, Li X, Yang WX. RGA analysis of the wheat leaf rust resistance gene Lr35. Acta Agriculturae Boreali Sinica. 2008;23(6):50-3.

53. Zhang J, Zhang QX, Cheng TR, Yang WR, Pan HT, Zhong JJ, et al. Highdensity genetic map construction and identification of a locus controlling weeping trait in an ornamental woody plant (Prunus mume Sieb. Et Zucc). DNA Res. 2015:22:1-9.

54. Li R, Li Y, Kristiansen K, Wang J. SOAP:short oligonucleotide alignment program. Bioinformatics. 2008;24:713-4.

55. Hill JT, Demarest BL, Bisgrove BW, Gorsi B, Su YC, Yost HJ. MMAPPR: mutation mapping analysis pipeline for pooled RNA-seq. Genome Res. 2013;23:687-97.

56. Cingolani P, Platts A, Wang LL, Coon N, Wang L, et al. A program for annotating and predicting the effects of single nucleotide polymorphisms, SnpEff:SNPs in the genome of Drosophila melanogaster strain w118; iso 2; iso 3. Fly. 2012;6:80-92.

57. Fekih $R$, Takagi $H$, Tamiru M, Abe A, Natsume S, Yaegashi $H$, et al. MutMap+:Genetic Mapping and Mutant Identification without Crossing in Rice. PLoS One. 2013;8:1-10. 
58. Takagi H, Abe A, Yoshida K, Kosugi S, Natsume S, Mitsuoka C, et al. QTLseq:Rapid mapping of quantitative trait loci in rice by whole genome resequencing of DNA from two bulked populations. Plant J. 2013;74:174-83.

59. Altschul SF, Madden TL, Schaffer AA, Zhang J, Zhang Z, Miller W, et al. Gapped BLAST and PSI-BLAST: a new generation of protein database search programs. Nucleic Acids Res. 1997;25:3389-402.

60. Deng YY, Li JQ, Wu SF, Zhu YP, Chen YW, He FC. Integrated nr database in protein annotation system and its localization. Comput En. 2006;32:71-4.

61. Livak KJ, Schmittgen TD. Analysis of relative gene expression data using realtime quantitative PCR and the $2^{-\triangle \Delta c t}$ method. Methods. 2001;25:402-8.

\section{Publisher's Note}

Springer Nature remains neutral with regard to jurisdictional claims in published maps and institutional affiliations.

- fast, convenient online submission

- thorough peer review by experienced researchers in your field

- rapid publication on acceptance

- support for research data, including large and complex data types

- gold Open Access which fosters wider collaboration and increased citations

- maximum visibility for your research: over $100 \mathrm{M}$ website views per year

At BMC, research is always in progress.

Learn more biomedcentral.com/submissions 